\title{
Diospyros montana Roxb.: A source of 1, 4-naphthoquinone dimers counting diospyrin esters
}

\author{
Venu Sharma \\ School of Biotechnology, Jammu University, Jammu, J\&K, India
}

\begin{abstract}
The heartwood and bark of Diospyros montana (Ebenaceae) were re-examined. Diospyrin, 3'methoxydiospyrin, diospyrin-2'-(epoxy-3-methyl-butanoate), diospyrin-2'-(2-hydroxypropanoate), diospyrin-3'(2-hydroxypropanoate) and tetrahydrodiospyrin were isolated from the plant and were identified on the basis of chromatographic and spectral analysis. Diospyrin-2'-(2-epoxy-3-methyl butanoate) was characterized by comparative study of $1 H$ NMR study of the other isolated diospyrin derivative analogues.
\end{abstract}

Keywords: Diospyros montana, diospyrin, diospyrin-2'-(2-epoxy-3-methyl butanoate), ester derivative, 1,4naphthoquinone dimmer

\section{Introduction}

Chemical examination of Ebenaceae generally confined to the genus Diospyros. A number of Diospyros species are reputed for their local herbal medicinal uses. In the treatment of asthma, abdominal pains, dysentry, leprosy, whopping cough, menstrual troubles and as antibiotics several parts of the plant have been used since a long time [1]. $50 \%$ Ethanol extract of D. montana and D.pereqrina were found to have weak antiprotozoal [2], antiviral and hypoglycemic activity. Different parts of D.montana Roxb. (bistendu ) are being used ethano-pharmacologically in the treatment of dysentry, hiccups, urinary stones and liver disorders [3]. Its leaves and seeds extract exhibited antibacterial activity [4]. Its various parts have been reported to be efficacious in fever, dysuria, gravel, neuralgia, pneumonia, puerperal fever and spider bite poison. Its bark extract has been reported to be used as anti inflammatory, antipyretic and analgesic. Alcoholic extract of its bark inhibited Ehrlich ascites carcinoma in mice [5]. Diospyros montana is a rich source of diospyrin 1 [4]. Heart wood and bark of this plant were re-examined. The present investigation reports the characterization of diospyrin esters; diospyrin-2 -(2-epoxy-3-methyl butanoate) 2, diospyrin-2'-(2hydroxypropanoate) 3, and diospyrin-3'-(2-hydroxypropanoate) 4 isolated from the acetone extract of heartwood and bark of Diospyros Montana. Diospyrin-2 -(2-epoxy-3-methyl butanoate) 2, is being reported for the first time.

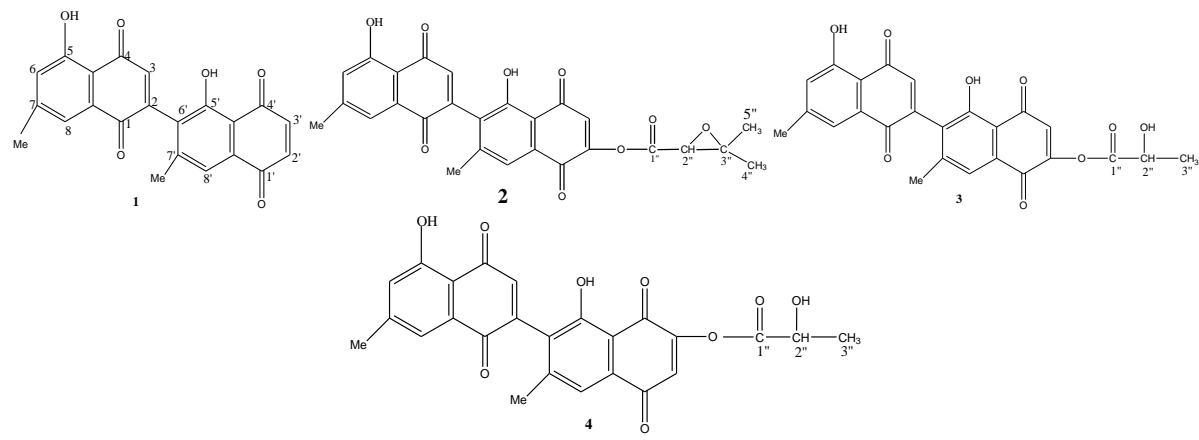

The new naphthoquinone dimer, diospyrin-2'-(2-epoxy-3-methyl butanoate) 2 was isolated as orange red crystals, m.p. $180-82^{\circ} \mathrm{C}$, Rf $0.15\left(\mathrm{CH}_{2} \mathrm{Cl}_{2}\right)$. It was characterized by its detailed spectral analysis. Its UV spectrum exhibited absorption bands at 220, 255 and $423 \mathrm{~nm}$ characteristic of a juglone moiety. Its IR spectrum in $\mathrm{KBr}$ revealed the presence of epoxy ring and ester functional group in the molecule by exhibiting absorption bands at 1250, 910 and $1745 \mathrm{~cm}^{-1}$. In addition bands at 1667 and $1644 \mathrm{~cm}^{-1}$ were also observed in its IR spectrum corresponding to unchelated and chelated carbonyl groups.

By analogy of its ${ }^{1} \mathrm{H}$ NMR spectrum with that of diospyrin, singlets at $\delta 2.35$ and 2.47 were ascribed to C-7' and C-7 methyl groups. Singlets observed in aromatic region at 7.53, 7.63 and 7.78 were assigned for H-6, H-8 and H-8' protons. H-3 olefinic proton resonated as singlet at 7.14 while 5-hydroxyl proton gave rise to another singlet at 11.91. The downfield shift of 5'-hydroxyl proton (12.49) in comparison to that of diospyrin(12.13) could be explained by placing an ester function at C-2' position. A singlet at $3.01 \mathrm{ppm}$ was observed due to epoxy proton. Another singlet at $1.56 \mathrm{ppm}$ integrated for $6 \mathrm{H}$ of two methyl group suggested that 2-epoxy-3-methylbutanoate moiety was present at C-2' position. A singlet at $6.94 \mathrm{ppm}$ integrated for one proton 
(H-3') provided further support for this assignment. Its CI mass spectrum exhibited an $[\mathrm{M}+1]^{+}$peak $\mathrm{m} / \mathrm{z} 489$ corresponding to its molecular formula $\mathrm{C}_{27} \mathrm{H}_{20} \mathrm{O}_{9}$.

${ }^{1} \mathrm{H}$ NMR spectra of $\mathbf{3}$ and $\mathbf{4}$ differ significantly only in the chemical shift of the signals associated with the hydroxyl proton at C-5'. In 4, it appeared relatively upfield (11.97) in comparison to that in $\mathbf{3}$ (12.46). This fact could be explained by placing the ester function at $\mathrm{C}-3$ position which shielded $\mathrm{C}-5$, hydroxyl proton [5]. ${ }^{1} \mathrm{H}$ NMR spectra were recorded on a Bruker DRX 300 instrument using $\mathrm{CDCl}_{3}$ as solvent.

These naphthoquinone derivatives of diospyrin displayed following spectral details:

Diospyrin (1): Orange red cubes, $\mathrm{R}_{\mathrm{f}} 0.43\left(\mathrm{CHCl}_{3}\right)$, m.p. $256-57^{\circ} \mathrm{C} . \mathrm{UV} \lambda_{\max } \mathrm{EtOH}(\log \varepsilon): 223$ (4.59), 254 (4.43), 438 (3.99) nm. IR $v_{\max } \mathrm{KBr}: 1669(\mathrm{C}=\mathrm{O}), 1645$ (chelated $\mathrm{C}=\mathrm{O}$ ), $1580 \mathrm{~cm}^{-1} .{ }^{1} \mathrm{H}$ NMR[90 $\left.\mathrm{MHz}, \mathrm{CDCl}_{3} \delta(\mathrm{ppm})\right]$ : 2.32s $\left(7^{\prime}-\mathrm{CH}_{3}\right), 2.46 \mathrm{~s}\left(7-\mathrm{CH}_{3}\right), 6.89 \mathrm{~s}(\mathrm{H}-3), 6.98 \mathrm{~s}\left(\mathrm{H}-2^{\prime}\right.$ and $\mathrm{H}-3$ ' $), 7.13 \mathrm{~d}(\mathrm{~J}=1 \mathrm{~Hz}, \mathrm{H}-6), 7.50 \mathrm{~d}(\mathrm{~J}=1 \mathrm{~Hz}, \mathrm{H}-8)$, 7.58s (H-8'), 11.88s (5-OH), 12.13s (5'-OH). CIMS (m/z, el.int.\%): $375[\mathrm{M}+1]^{+} \mathrm{C}_{22} \mathrm{H}_{14} \mathrm{O}_{6}(100), 357$ [375$\left.\mathrm{H}_{2} \mathrm{O}\right]^{+}$.

Diospyrin-2'-(2-epoxy-3-methyl butanoate) (2): Orange red crystals, $\mathrm{Rf} 0.15\left(\mathrm{CH}_{2} \mathrm{Cl} 2\right)$, m.p.180-82 ${ }^{0} . \mathrm{UV} \lambda_{\max }$ EtoAc(loge): 220(4.23), 255(4.18), 423(3.60) nm. IR $v_{\text {max. }} \mathrm{KBr}$ : 1745 (esterC=O), 1667 (unchelated carbonyl), 1644 (chelated carbonyl), 1250 (C-O-C unsymmetrical stretching), 910 (symm. str.). ${ }^{1} \mathrm{H}$ NMR [30 MHz, $\mathrm{CDCl}_{3}$,

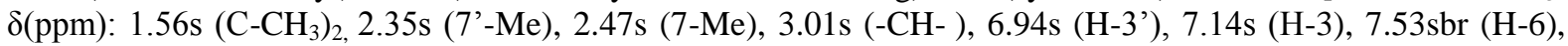
7.63sbr (H-8), 7.78s (H-8'), 11.91s (5-OH), 12.49s (5'-OH). CIMS (m/z); 489[M+1] ${ }^{+}, \mathrm{C}_{27} \mathrm{H}_{20} \mathrm{O}_{9}, 474$ [489$\left.\mathrm{CH}_{3}\right]^{+}, 456\left[474-\mathrm{H}_{2} \mathrm{O}\right]^{+}$etc.

Diospyrin-2'-(2-hydroxypropanoate) (3): Dark red crystals, $\mathrm{Rf} 0.70\left(\mathrm{CH}_{2} \mathrm{Cl}_{2}\right)$.

UV $\lambda_{\max } \operatorname{EtoAc}(\log \varepsilon): 220(4.15), 255(4.17), 423(3.53) \mathrm{nm}$. IR $v_{\max } \mathrm{KBr}$ : 3440-3400 (OH), 1745 (ester $\left.\mathrm{C}=\mathrm{O}\right)$, 1667 (unchelated carbonyl), 1644 (chelated carbonyl), $1575 \mathrm{~cm}^{-1} .{ }^{1} \mathrm{H}$ NMR $\left[30 \mathrm{MHz}, \mathrm{CDCl}_{3}, \delta(\mathrm{ppm}): 1.43 \mathrm{~d}\right.$ $\left(\mathrm{J}=7 \mathrm{~Hz}, \mathrm{C}-\mathrm{CH}_{3}\right)_{2}, 2.30 \mathrm{~s}\left(7^{\prime}-\mathrm{Me}\right), 2.48 \mathrm{~s}(7-\mathrm{Me}), 3.17 \mathrm{q}(\mathrm{J}=7 \mathrm{~Hz},-\mathrm{CH}-\mathrm{O}), 6.54 \mathrm{~s}\left(\mathrm{H}_{-3}{ }^{\prime}\right), 6.92 \mathrm{~s}(\mathrm{H}-3), 7.14 \mathrm{~s}(\mathrm{H}-6)$, 7.52s (H-8), 7.56s (H-8'), 11.90s (5-OH), 12.46s (5'-OH). CIMS (m/z, rel.int.\%); 463[M+1] $]^{+}, \mathrm{C}_{25} \mathrm{H}_{18} \mathrm{O}_{9}(100)$, $445\left[463-\mathrm{H}_{2} \mathrm{O}\right]^{+}$etc.

Diospyrin-3'- (2-hydroxypropanoate) (4): Red crystals, $\mathrm{Rf} 0.63\left(\mathrm{CH}_{2} \mathrm{Cl}_{2}\right)$.

UV $\lambda_{\max } \operatorname{EtoAc}(\log \varepsilon): 248(4.35), 293(4.20), 425(3.95) \mathrm{nm}$. IR $v_{\max }$ KBr: 3460 (broad, OH), 1742 (ester $\mathrm{C}=\mathrm{O}$ ), 1665 (unchelated carbonyl), 1645 (chelated carbonyl), $\mathrm{cm}^{-1} .{ }^{1} \mathrm{H}$ NMR $\left[30 \mathrm{MHz}, \mathrm{CDCl}_{3}, \delta(\mathrm{ppm}): 1.43 \mathrm{~d}(\mathrm{~J}=7 \mathrm{~Hz}\right.$, ${\left.\mathrm{C}-\mathrm{CH}_{3}\right)}_{3} 2.31 \mathrm{~s}\left(7^{\prime}-\mathrm{Me}\right), 2.45 \mathrm{~s}(7-\mathrm{Me}), 3.18 \mathrm{q}(\mathrm{J}=7 \mathrm{~Hz},-\mathrm{CH}-\mathrm{O}), 6.61 \mathrm{~s}\left(\mathrm{H}^{\prime}{ }^{\prime}\right), 6.89 \mathrm{~s}(\mathrm{H}-3), 7.13 \mathrm{sbr}(\mathrm{H}-6), 7.52 \mathrm{sbr}$ $(\mathrm{H}-8), 7.58 \mathrm{~s}\left(\mathrm{H}-8^{\prime}\right), 11.88 \mathrm{~s}(5-\mathrm{OH}), 11.97 \mathrm{~s}\left(5^{\prime}-\mathrm{OH}\right)$. CIMS (m/z, rel.int.\%); 463[M+1] $]^{+}, \mathrm{C}_{25} \mathrm{H}_{18} \mathrm{O}_{9}(100), 435$ $[463-\mathrm{CO}]^{+}, 407[435-\mathrm{CO}]^{+}$etc.

\section{Acknowledgement}

The author would like to acknowledge Prof. Pahup Singh, Department of Chemistry, University of Rajasthan, Jaipur for his guidance and providing all necessary facilities for the research work.

\section{References}

[1]. G. K. A. S. S. Narayan, L. R. Row and P. Satyanarayana, Curr. Sci., 47, 345 (1978)

[2]. $\quad$ V.S.S. Kanamreddi and W.W. Colin, Evid Based Complement Alternat Med. 5 (2) , 187 (2008).

[3]. M. G. Jayathritha, B. K. Shah and K. N. Patel, Journal of Medicinal and Aromatic Plant Sciences, 22, 29 (2000)

[4]. M. P. Gautam and R. M. Purohit, Indian J. Pharm., 35, 93 (1973).

[5]. L. V. Asolkar, K. K. Kakkar, O. J. Chakre, Second Supplement to Glossary of Indian Medicinal Plants with Active principles, CSIR, New Delhi, Part-1, 279 (1965). 